CRYSTALLOGRAPHIC COMMUNICATIONS
Received 5 September 2018

Accepted 19 September 2018

Edited by D.-J. Xu, Zhejiang University (Yuquan Campus), China

\section{Crystal structure and Hirshfeld surface analysis and of 2-ammoniumylmethyl-1H-benzimidazol-3-ium chloride monohydrate. Corrigendum}

Pinar Sen, ${ }^{a}$ Sevgi Kansiz, ${ }^{b}$ Necmi Dege, ${ }^{b}$ S. Zeki Yildiz ${ }^{c}$ and Galyna G. Tsapyuk ${ }^{d *}$

${ }^{a}$ Uskudar University, Faculty of Engineering and Natural Sciences, Department of Forensic Science, 34662, Istanbul, Turkey, ${ }^{\mathbf{b}}$ Ondokuz Mayıs University, Faculty of Arts and Sciences, Department of Physics, 55139, Kurupelit, Samsun, Turkey, 'Sakarya University, Faculty of Arts and Sciences, Department of Chemistry, 54187 Sakarya, Turkey, and ${ }^{\mathbf{d}}$ Taras Shevchenko National University of Kyiv, Department of Chemistry, 64 Vladimirska Str., Kiev 01601, Ukraine.

*Correspondence e-mail: tsapyuk@ukr.net

In the paper by Sen et al. [Acta Cryst. (2018), E74, 1517-1520], the address of the correspondence author is incorrect.

In the paper by Sen et al. (2018), the address of the correspondence author, Galyna G. Tsapyuk, should be 'Taras Shevchenko National University of Kyiv, Department of Chemistry, 64 Vladimirska Str., Kiev 01601, Ukraine', as given above.

\section{References}

Sen, P., Kansiz, S., Dege, N., Yildiz, S. Z. \& Tsapyuk, G. G. (2018). Acta Cryst. E74, 1517-1520. 
CRYSTALLOGRAPHIC COMMUNICATIONS

ISSN 2056-9890

Received 5 September 2018

Edited by D.-J. Xu, Zhejiang University (Yuquan Campus), China

Keywords: crystal structure; imidazol; ethanaminium; ethanaminium chloride; Hirshfeld surface.

CCDC reference: 1868580

Supporting information: this article has supporting information at journals.iucr.org/e
Accepted 19 September 2018

\section{Crystal structure and Hirshfeld surface analysis and of 2-ammoniumylmethyl-1H-benzimidazol-3-ium chloride monohydrate}

\author{
Pinar Sen, ${ }^{\text {a Sevgi Kansiz, }}{ }^{\mathrm{b}}$ Necmi Dege, ${ }^{\mathrm{b}}$ S. Zeki Yildiz ${ }^{\mathrm{c}}$ and Galyna G. Tsapyuk ${ }^{\mathrm{d} *}$ \\ a Uskudar University, Faculty of Engineering and Natural Sciences, Department of Forensic Science, 34662, Istanbul, \\ Turkey, 'b Ondokuz Mayıs University, Faculty of Arts and Sciences, Department of Physics, 55139, Kurupelit, Samsun, \\ Turkey, 'Sakarya University, Faculty of Arts and Sciences, Department of Chemistry, 54187 Sakarya, Turkey, and \\ dDepartment of General Chemistry, O. O. Bohomolets National Medical University, Shevchenko Blvd. 13, 01601 Kiev, \\ Ukraine. *Correspondence e-mail: tsapyuk@ukr.net
}

The asymmetric unit of the title compound, $\mathrm{C}_{8} \mathrm{H}_{11} \mathrm{~N}_{3}{ }^{2+} \cdot 2 \mathrm{Cl}^{-} \cdot \mathrm{H}_{2} \mathrm{O}$, contains three organic cations, six chloride anions and three water molecules of crystallization, which are connected by extensive hydrogen-bonding interactions into a threedimensional supramolecular architecture. Hirshfeld surface analysis and twodimensional fingerprint plots indicate that the most important contributions to the crystal packing are from $\mathrm{H} \cdots \mathrm{H}(37.4 \%), \mathrm{Cl} \cdots \mathrm{H} / \mathrm{H} \cdots \mathrm{Cl}(35.5 \%), \mathrm{C} \cdots \mathrm{H} /$ $\mathrm{H} \cdot \mathrm{C}(9.5 \%)$ and $\mathrm{C} \cdot \mathrm{C}(6.9 \%)$ interactions.

\section{Chemical context}

Heterocyclic compounds containing nitrogen such as benzimidazoles and their derivatives have attracted attention because of their medicinal applications as antiulcer, anticancer, antifungal, antimycobacterial and anti-inflammatory agents (El-masry et al., 2000). Besides being important pharmacophores, in particular amine-substituted benzimidazoles are good intermediates for the synthesis of different organic compounds (Maurya et al., 2007). General methods for the preparation of benzimidazoles involve the reaction of $o$-phenylenediamine and carboxylic acid or its derivatives under harsh dehydrating conditions or with aldehydes followed by oxidation (Peng et al., 2014).
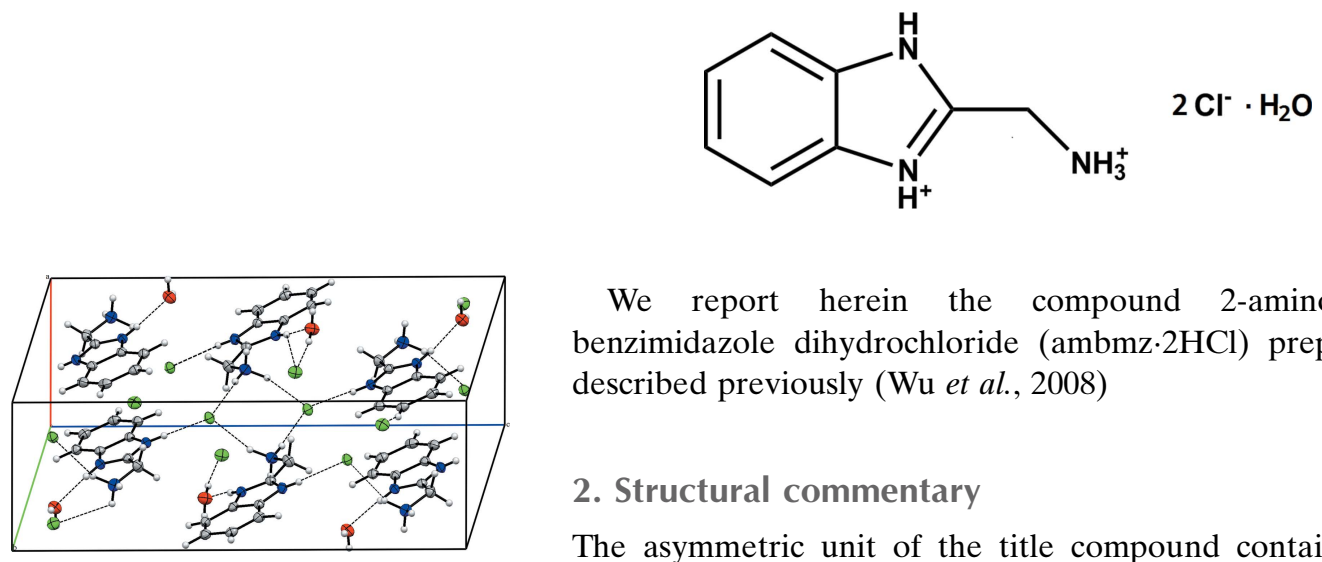

We report herein the compound 2-aminomethylbenzimidazole dihydrochloride $($ ambmz $\cdot 2 \mathrm{HCl})$ prepared as described previously (Wu et al., 2008)

\section{Structural commentary}

The asymmetric unit of the title compound contains three organic cations, six chloride anions and three water molecules of crystallization, which are connected by $\mathrm{O}-\mathrm{H} \cdots \mathrm{Cl}, \mathrm{N}-$ $\mathrm{H} \cdots \mathrm{O}$ and $\mathrm{N}-\mathrm{H} \cdots \mathrm{Cl}$ hydrogen bonds (Fig. 1). The r.m.s. deviations of the benzimidazolium ring systems are $0.0085 \AA$ 
Table 1

Hydrogen-bond geometry $\left(\AA,^{\circ}\right)$.

\begin{tabular}{|c|c|c|c|c|}
\hline$D-\mathrm{H} \cdots A$ & $D-\mathrm{H}$ & $\mathrm{H} \cdots A$ & $D \cdots A$ & $\overline{D-\mathrm{H} \cdots A}$ \\
\hline $\mathrm{O} 1-\mathrm{H} 1 A \cdots \mathrm{Cl} 4^{\mathrm{i}}$ & $0.82(2)$ & $2.63(2)$ & $3.4431(15)$ & $168(3)$ \\
\hline $\mathrm{O} 1-\mathrm{H} 2 B \cdots \mathrm{Cl} 2$ & $0.81(2)$ & $2.36(2)$ & $3.1598(18)$ & $169(3)$ \\
\hline $\mathrm{O} 2-\mathrm{H} 2 C \cdots \mathrm{Cl} 3$ & $0.82(2)$ & $2.33(2)$ & 3.1244 (17) & $165(2)$ \\
\hline $\mathrm{O} 2-\mathrm{H} 2 D \cdots \mathrm{Cl} 1$ & $0.81(1)$ & $2.64(1)$ & $3.4492(15)$ & $171(3)$ \\
\hline $\mathrm{O} 3-\mathrm{H} 3 D \cdots \mathrm{Cl} 5^{\mathrm{ii}}$ & $0.80(2)$ & $2.69(2)$ & $3.4586(15)$ & $164(3)$ \\
\hline $\mathrm{O} 3-\mathrm{H} 3 E \cdots \mathrm{Cl}^{\mathrm{ii}}$ & $0.82(2)$ & $2.32(2)$ & $3.1364(18)$ & $173(3)$ \\
\hline $\mathrm{N} 1-\mathrm{H} 1 \cdots \mathrm{O} 1$ & $0.83(2)$ & $1.92(2)$ & $2.746(2)$ & $174(2)$ \\
\hline $\mathrm{N} 2-\mathrm{H} 2 \cdots \mathrm{Cl} 1^{\mathrm{iii}}$ & $0.71(2)$ & $2.44(2)$ & 3.1519 (17) & $175(2)$ \\
\hline $\mathrm{N} 3-\mathrm{H} 3 \cdots \mathrm{Cl} 3$ & $0.88(3)$ & $2.30(3)$ & $3.105(2)$ & $153(2)$ \\
\hline $\mathrm{N} 3-\mathrm{H} 3 A \cdots \mathrm{Cl} 1$ & $0.86(4)$ & $2.26(3)$ & $3.119(2)$ & $173(3)$ \\
\hline $\mathrm{N} 3-\mathrm{H} 3 B \cdots \mathrm{Cl} 4^{\mathrm{iv}}$ & $0.99(3)$ & $2.33(2)$ & $3.267(2)$ & $156.8(19)$ \\
\hline $\mathrm{N} 4-\mathrm{H} 4 A \cdots \mathrm{O} 2$ & $0.85(2)$ & $1.91(2)$ & $2.754(2)$ & $171(2)$ \\
\hline $\mathrm{N} 5-\mathrm{H} 5 A \cdots \mathrm{Cl} 5$ & $0.83(2)$ & $2.31(2)$ & $3.1205(17)$ & $165(2)$ \\
\hline $\mathrm{N} 6-\mathrm{H} 6 A \cdots \mathrm{Cl} 2$ & $0.87(3)$ & $2.33(3)$ & 3.107 (2) & $150(2)$ \\
\hline $\mathrm{N} 6-\mathrm{H} 6 B \cdots \mathrm{Cl}^{\mathrm{v}}$ & $0.98(3)$ & $2.36(2)$ & $3.287(2)$ & $158.2(18)$ \\
\hline $\mathrm{N} 6-\mathrm{H} 6 C \cdots \mathrm{Cl} 4^{\mathrm{i}}$ & $0.79(3)$ & $2.35(4)$ & 3.1347 (19) & $176(3)$ \\
\hline $\mathrm{N} 7-\mathrm{H} 7 \ldots \mathrm{O} 3$ & $0.85(3)$ & $1.89(3)$ & $2.742(2)$ & $175(3)$ \\
\hline 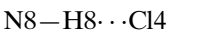 & $0.81(2)$ & $2.31(2)$ & $3.1049(17)$ & $172(2)$ \\
\hline $\mathrm{N} 9-\mathrm{H} 9 A \cdots \mathrm{Cl} 6$ & $0.83(3)$ & $2.35(3)$ & $3.100(2)$ & $151(2)$ \\
\hline $\mathrm{N} 9-\mathrm{H} 9 B \cdots \mathrm{Cl} 5$ & $0.80(3)$ & $2.32(2)$ & $3.120(2)$ & $176(2)$ \\
\hline $\mathrm{N} 9-\mathrm{H} 9 C \cdots \mathrm{Cl}^{\mathrm{vi}}$ & 0.99 (3) & $2.34(3)$ & $3.270(2)$ & $157(2)$ \\
\hline $\mathrm{C} 4-\mathrm{H} 4 \cdots \mathrm{Cl} 1^{\mathrm{i}}$ & $0.92(2)$ & $2.81(2)$ & $3.535(2)$ & $136.7(16)$ \\
\hline $\mathrm{C} 8-\mathrm{H} 8 B \cdots \mathrm{Cl}^{2 \mathrm{vi}}$ & $0.93(3)$ & $2.65(3)$ & $3.544(2)$ & $162(2)$ \\
\hline
\end{tabular}

Symmetry codes: (i) $x, y-1, z$; (ii) $-x,-y+1,-z+1$; (iii) $-x+3,-y,-z+2$; (iv) $x+1, y-1, z$; (v) $x-1, y, z$; (vi) $-x+1,-y+1,-z+1$; (vii) $-x+2,-y,-z+2$.

for $\mathrm{N} 1 / \mathrm{N} 2 / \mathrm{C} 1-\mathrm{C} 7,0.0076 \AA$ for N4/N5/C9-C15, $0.0063 \AA$ for $\mathrm{N} 7 / \mathrm{N} 8 / \mathrm{C} 17-\mathrm{C} 23$ with maximum deviations from planarity of 0.0169 (13) $\AA$ for atom C7, 0.0149 (13) $\AA$ for atom C15 and 0.0132 (13) A for atom C23, respectively. The observed bond lengths are in good agreement with previously reported values (Cui, 2011).

\section{Supramolecular features}

The crystal packing of the title compound features extensive hydrogen bonding (Table 1 and Fig. and 2) involving all three $\mathrm{O}$ atoms and all nine $\mathrm{N}$ atoms. $\mathrm{N} 5-\mathrm{H} 5 A \cdots \mathrm{Cl} 5, \mathrm{~N} 8-$ $\mathrm{H} 8 \cdots \mathrm{Cl} 4, \quad \mathrm{~N} 2-\mathrm{H} 2 \cdots \mathrm{Cl} 1^{\mathrm{ii}}, \quad \mathrm{N} 9-\mathrm{H} 9 C \cdot \cdots \mathrm{Cl} 5^{\mathrm{vi}}$ and $\mathrm{N} 6-$ $\mathrm{H} 6 B \cdots \mathrm{Cl} 1^{\mathrm{v}}$ hydrogen bonds link the ions into chains along the $c$-axis direction. These chains are linked by $\mathrm{O}-\mathrm{H} \cdots \mathrm{Cl}$ and $\mathrm{N}-$ $\mathrm{H}$... O hydrogen bonds, generating a three-dimensional network (Fig. 2).

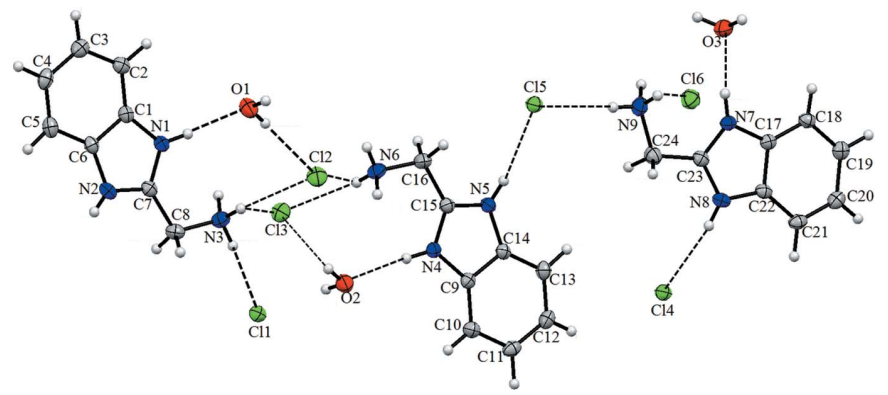

Figure 1

The molecular structure of the title compound, showing the atom labelling. Displacement ellipsoids are drawn at the $20 \%$ probability level.

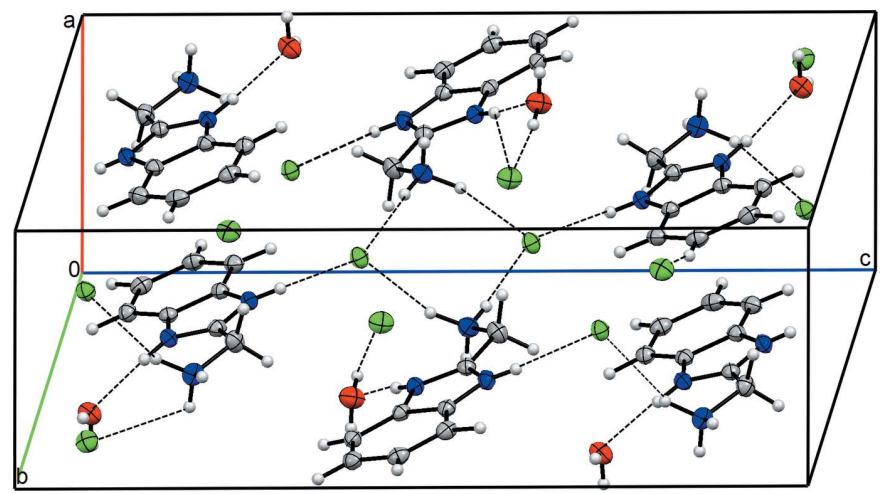

Figure 2

The view of the crystal packing of the title compound. Dashed lines denote the hydrogen bonds.

\section{Hirshfeld surface analysis}

The Hirshfeld surface analysis was performed using Crystal Explorer (Turner et al., 2017). The Hirshfeld surfaces, illustrated in Fig. 3, and their associated two-dimensional fingerprint plots were used to quantify the various intermolecular
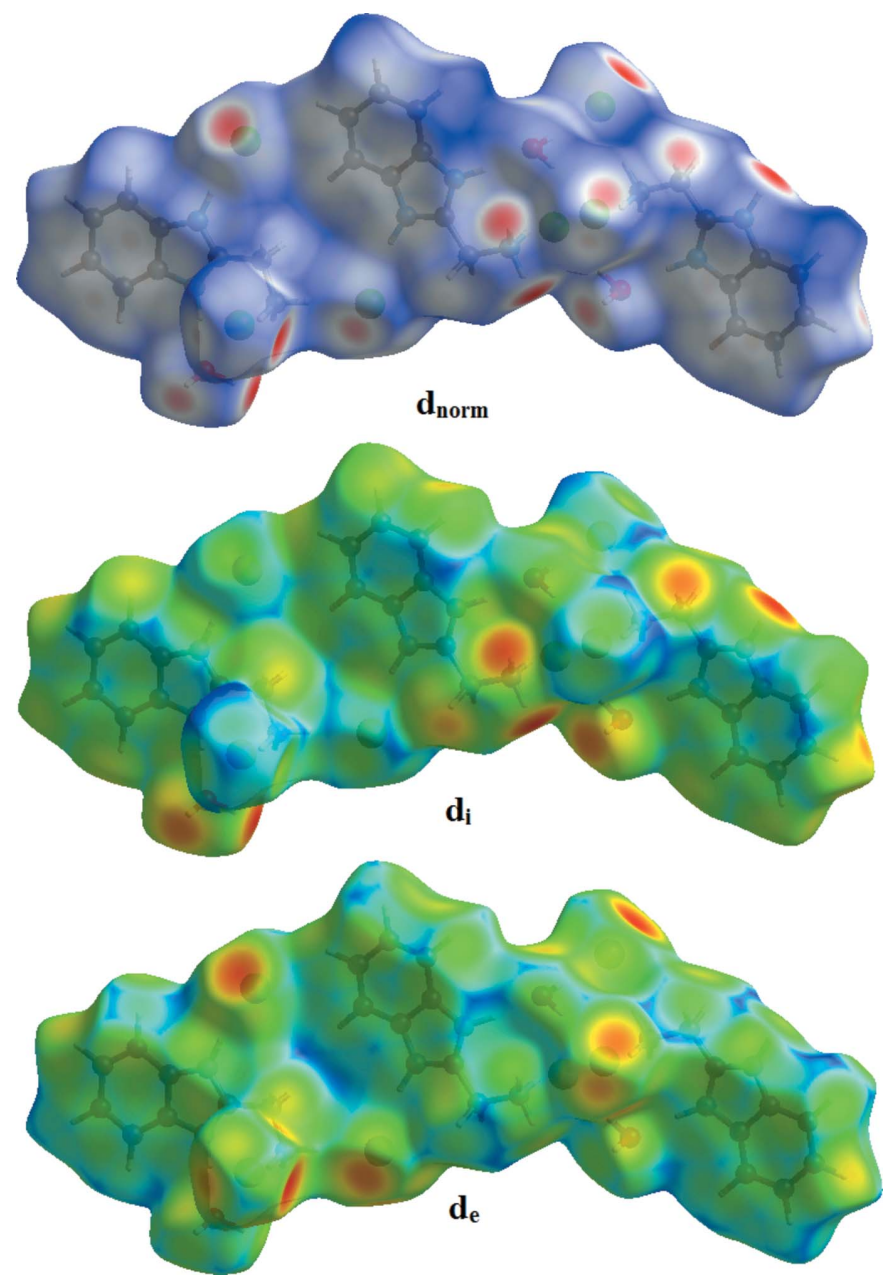

Figure 3

The Hirshfeld surface of the title compound mapped over $d_{\text {norm }}, d_{\mathrm{i}}$ and $d_{\mathrm{e}}$. 


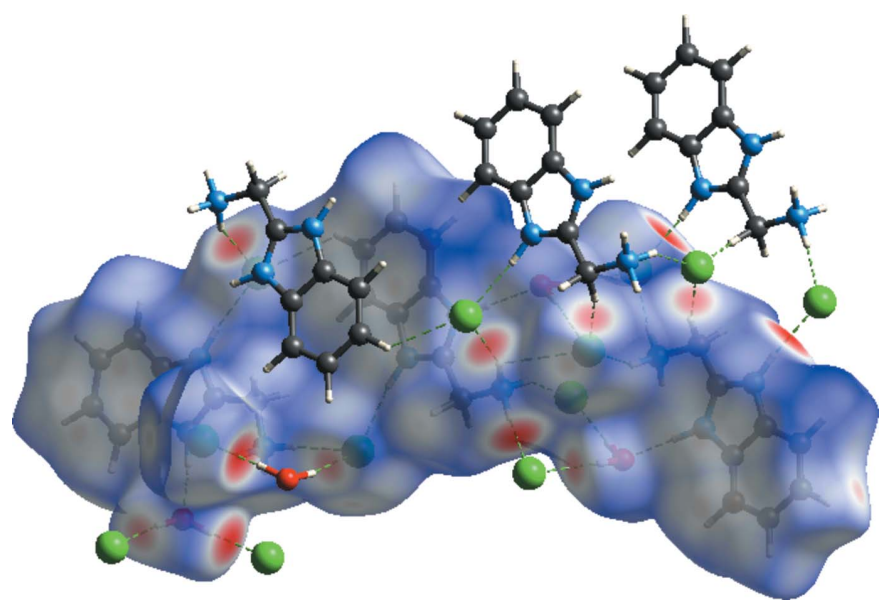

Figure 4

Hirshfeld surface mapped over $d_{\text {norm }}$ to visualize the intermolecular interactions of the title compound.

interactions in the synthesized complex. Red spots on the Hirshfeld surfaces indicate the intermolecular contacts involved in strong hydrogen bonds and interatomic contacts (Gümüş et al., 2018; Kansız et al., 2018; Kansız \& Dege, 2018). The red spots in Fig. 4 correspond to the $\mathrm{H} \cdots \mathrm{Cl}$ contacts resulting from the $\mathrm{N}-\mathrm{H} \cdots \mathrm{Cl}$ and $\mathrm{O}-\mathrm{H} \cdots \mathrm{Cl}$ hydrogen bonds. The Hirshfeld surfaces were mapped using a standard (high) surface resolution with the three-dimensional $d_{n o r m}$ surfaces mapped over a fixed colour scale of -0.518 (red) to 1.174 (blue) a.u..

Fig. 5 shows the two-dimensional fingerprint plot of all the contacts contributing to the Hirshfeld surface represented in normal mode. Fig. 6 shows the two-dimensional fingerprint plots of the $\left(d_{\mathrm{i}}, d_{\mathrm{e}}\right)$ points associated with various atoms. $\mathrm{H} \cdots \mathrm{H}$ contacts contribute $37.4 \%$ to the Hirshfeld surface. The graph for $\mathrm{Cl} \cdots \mathrm{H} / \mathrm{H} \cdots \mathrm{Cl}$ shows the contacts between the chlorine atoms inside the Hirshfeld surface and the hydrogen atoms outside the surface and vice versa, and has two symmetrical wings on the left and right sides (35.5\%). Further, there are $\mathrm{C} \cdots \mathrm{H} / \mathrm{H} \cdots \mathrm{C}(9.5 \%), \mathrm{C} \cdots \mathrm{C}(6.9 \%), \mathrm{O} \cdots \mathrm{H} / \mathrm{H} \cdots \mathrm{O}$ $(4.1 \%)$ and $\mathrm{N} \cdots \mathrm{H} / \mathrm{H} \cdots \mathrm{N}(3.4 \%)$ contacts.

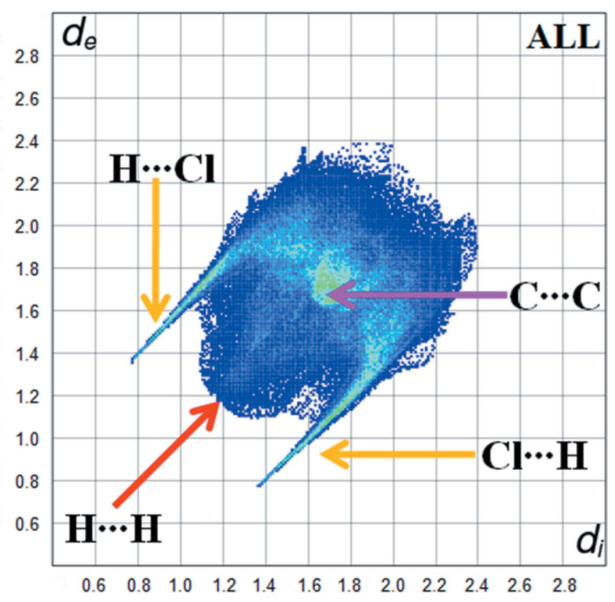

Figure 5

Fingerprint plot of all the contacts.

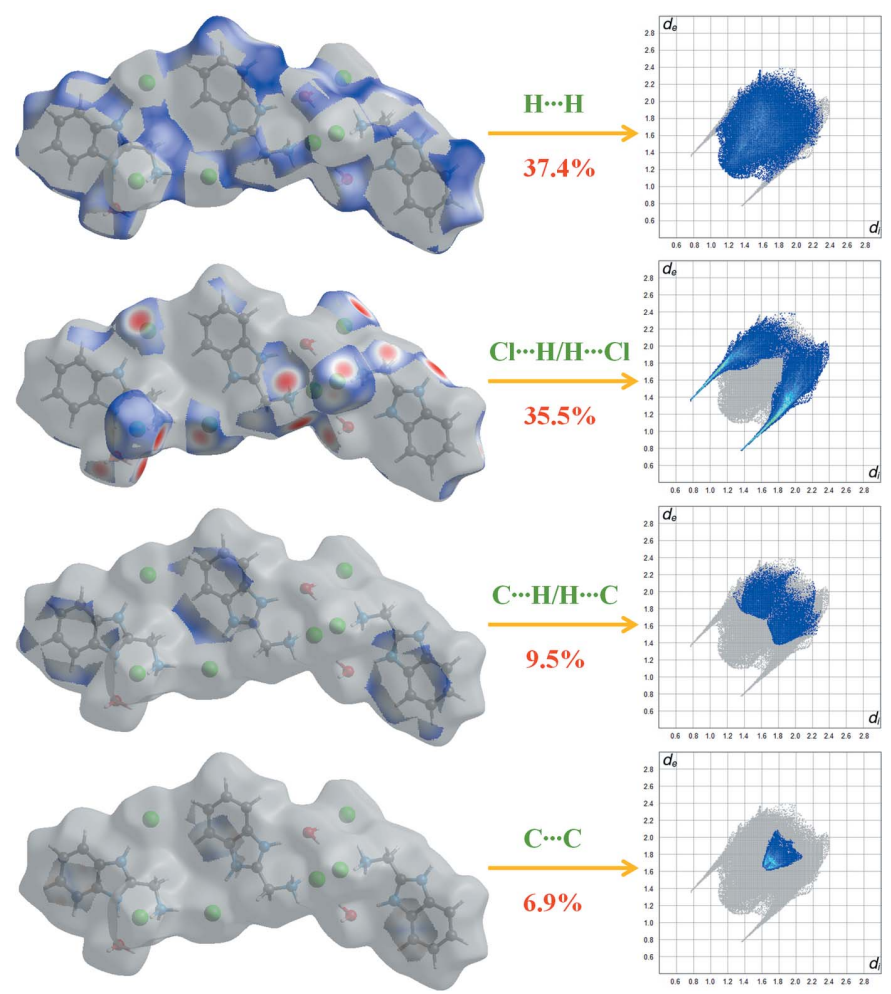

Figure 6

Two-dimensional fingerprint plots with a $d_{\text {norm }}$ view of the $\mathrm{H} \cdots \mathrm{H}$ $(37.4 \%), \mathrm{Cl} \cdots \mathrm{H} / \mathrm{H} \cdots \mathrm{Cl}(35.5 \%), \mathrm{C} \cdots \mathrm{H} / \mathrm{H} \cdots \mathrm{C}(9.5 \%)$ and $\mathrm{C} \cdots \mathrm{C}(6.9 \%)$ contacts in the title compound.

\section{Synthesis and crystallization}

$o$-Phenylenediamine $(10.8 \mathrm{~g}, 99.87 \mathrm{mmol})$ and glycine $(10.00 \mathrm{~g}$, $133.2 \mathrm{mmol})$ were dissolved in $5.5 \mathrm{M} \mathrm{HCl}(150 \mathrm{~mL})$. The reaction mixture was purged by argon at room temperature and heated up to reflux temperature for $12 \mathrm{~h}$. The reaction was monitored by TLC. After completion of the reaction, the mixture was concentrated to $50 \mathrm{~mL}$ and kept at $269 \mathrm{~K}$ for $2 \mathrm{~d}$. The crystals were filtered off and washed twice with acetone and dried to give the desired product (Fig. 7).

\section{Refinement}

Crystal data, data collection and structure refinement details are summarized in Table 2. C-bound $\mathrm{H}$ atoms were positioned geometrically with $\mathrm{C}-\mathrm{H}$ distances of $0.93-0.97 \AA$. and refined as riding, with $U_{\text {iso }}(\mathrm{H})=1.2 U_{\text {eq }}(\mathrm{C})$. N-bound $\mathrm{H}$ atoms were located in difference-Fourier maps and refined isotropically. The coordinates of the water $\mathrm{H}$ atoms were determined from a difference-Fourier map and refined isotropically subject to a restraint of $\mathrm{O}-\mathrm{H}=0.82(4) \AA$.<smiles>Nc1ccccc1N</smiles><smiles>NCC(=O)O</smiles><smiles>NCc1nc2ccccc2[nH]1</smiles>

Figure 7

The synthesis of the title compound. 
Table 2

Experimental details.

\begin{tabular}{|c|c|}
\hline \multicolumn{2}{|l|}{ Crystal data } \\
\hline Chemical formula & $\mathrm{C}_{8} \mathrm{H}_{11} \mathrm{~N}_{3}^{+} \cdot 2 \mathrm{Cl}^{-} \cdot \mathrm{H}_{2} \mathrm{O}$ \\
\hline$M_{\mathrm{r}}$ & 238.11 \\
\hline Crystal system, space group & Triclinic, $P \overline{1}$ \\
\hline Temperature (K) & 296 \\
\hline$a, b, c(\AA)$ & $\begin{array}{l}6.9340(4), 12.1198(7) \\
\quad 19.2128(11)\end{array}$ \\
\hline$\alpha, \beta, \gamma\left(^{\circ}\right)$ & $99.859(5), 90.647(5), 90.247(5)$ \\
\hline$V\left(\AA^{3}\right)$ & $1590.64(16)$ \\
\hline$Z$ & 6 \\
\hline Radiation type & Mo $K \alpha$ \\
\hline$\mu\left(\mathrm{mm}^{-1}\right)$ & 0.58 \\
\hline Crystal size $(\mathrm{mm})$ & $0.57 \times 0.50 \times 0.46$ \\
\hline \multicolumn{2}{|l|}{ Data collection } \\
\hline Diffractometer & Stoe IPDS 2 \\
\hline Absorption correction & $\begin{array}{l}\text { Integration }(X-R E D 32 ; \text { Stoe } \& \text { Cie, } \\
\text { 2002) }\end{array}$ \\
\hline$T_{\min }, T_{\max }$ & $0.788,0.828$ \\
\hline $\begin{array}{l}\text { No. of measured, independent and } \\
\text { observed }[I>2 \sigma(I)] \text { reflections }\end{array}$ & $16045,6254,5000$ \\
\hline$R_{\text {int }}$ & 0.064 \\
\hline$(\sin \theta / \lambda)_{\max }\left(\AA^{-1}\right)$ & 0.617 \\
\hline \multicolumn{2}{|l|}{ Refinement } \\
\hline$R\left[F^{2}>2 \sigma\left(F^{2}\right)\right], w R\left(F^{2}\right), S$ & $0.035,0.092,0.96$ \\
\hline No. of reflections & 6254 \\
\hline No. of parameters & 536 \\
\hline No. of restraints & 9 \\
\hline $\mathrm{H}$-atom treatment & All H-atom parameters refined \\
\hline$\Delta \rho_{\max }, \Delta \rho_{\min }\left(\mathrm{e} \AA^{-3}\right)$ & $0.34,-0.28$ \\
\hline
\end{tabular}

Computer programs: $X$-AREA and X-RED32 (Stoe \& Cie, 2002), SHELXL2017/1 (Sheldrick, 2015), ORTEP-3 for Windows and WinGX (Farrugia, 2012) and PLATON (Spek, 2009).

\section{Acknowledgements}

The authors acknowledge the Faculty of Arts and Sciences, Ondokuz Mayis University, Turkey, for the use of the Stoe IPDS 2 diffractometer (purchased under grant F.279 of the University Research Fund).

\section{References}

Cui, Y. (2011). Acta Cryst. E67, o625-o626.

El-masry, A. H., Fahmy, H. H. \& Ali Abdelwahed, S. (2000). Molecules, 5, 1429-1438.

Farrugia, L. J. (2012). J. Appl. Cryst. 45, 849-854.

Gümüş, M. K., Kansız, S., Aydemir, E., Gorobets, N. Y. \& Dege, N. (2018). J. Mol. Struct. 1168, 280-290.

Kansiz, S., Almarhoon, Z. M. \& Dege, N. (2018). Acta Cryst. E74, 217-220.

Kansız, S. \& Dege, N. (2018). J. Mol. Struct. 1173, 42-51.

Maurya, M. R., Chandrakar, A. K. \& Chand, S. (2007). J. Mol. Catal. A Chem. 263, 227-237.

Peng, P., Xiong, J., Mo, G., Zheng, J., Chen, R., Chen, X. \& Wang, Z. (2014). Amino Acids, 46, 2427-2433.

Sheldrick, G. M. (2015). Acta Cryst. C71, 3-8.

Spek, A. L. (2009). Acta Cryst. D65, 148-155.

Stoe \& Cie (2002). $X$-AREA and $X$-RED32. Stoe \& Cie GmbH, Darmstadt, Germany.

Turner, M. J., MacKinnon, J. J., Wolff, S. K., Grimwood, D. J., Spackman, P. R., Jayatilaka, D. \& Spackman, M. A. (2017). Crystal Explorer17.5. University of Western Australia, Perth.

Wu, H.-Y., Li, H., Zhu, B.-L., Wang, S.-R., Zhang, S.-M., Wu, S.-H. \& Huang, W.-P. (2008). Transition Met. Chem. 33, 9-15. 


\section{supporting information}

Acta Cryst. (2018). E74, 1517-1520］[https://doi.org/10.1107/S205698901801335X]

Crystal structure and Hirshfeld surface analysis and of 2-ammoniumylmethyl)-1H-benzimidazol-3-ium chloride monohydrate

Pinar Sen, Sevgi Kansiz, Necmi Dege, S. Zeki Yildiz and Galyna G. Tsapyuk

Computing details

Data collection: $X$-AREA (Stoe \& Cie, 2002); cell refinement: $X$-AREA (Stoe \& Cie, 2002); data reduction: $X$ - $R E D 32$

(Stoe \& Cie, 2002); program(s) used to solve structure: WinGX (Farrugia, 2012); program(s) used to refine structure:

SHELXL2017/1 (Sheldrick, 2015); molecular graphics: ORTEP-3 for Windows (Farrugia, 2012); software used to prepare material for publication: WinGX (Farrugia, 2012) and PLATON (Spek, 2009).

2-Ammoniumylmethyl-1 H-benzimidazol-3-ium chloride monohydrate

Crystal data

$\mathrm{C}_{8} \mathrm{H}_{11} \mathrm{~N}_{3}{ }^{+} \cdot 2 \mathrm{Cl}^{-} \cdot \mathrm{H}_{2} \mathrm{O} \cdot$

$M_{r}=238.11$

Triclinic, $P \overline{1}$

$a=6.9340(4) \AA$

$b=12.1198(7) \AA$

$c=19.2128(11) \AA$

$\alpha=99.859(5)^{\circ}$

$\beta=90.647(5)^{\circ}$

$\gamma=90.247(5)^{\circ}$

$V=1590.64(16) \AA^{3}$

\section{Data collection}

Stoe IPDS 2 diffractometer

Radiation source: sealed X-ray tube, 12 × 0.4 $\mathrm{mm}$ long-fine focus

Detector resolution: 6.67 pixels $\mathrm{mm}^{-1}$

rotation method scans

Absorption correction: integration

(X-RED32; Stoe \& Cie, 2002)

$T_{\min }=0.788, T_{\max }=0.828$

\section{Refinement}

Refinement on $F^{2}$

Least-squares matrix: full

$R\left[F^{2}>2 \sigma\left(F^{2}\right)\right]=0.035$

$w R\left(F^{2}\right)=0.092$

$S=0.96$

6254 reflections

536 parameters

9 restraints

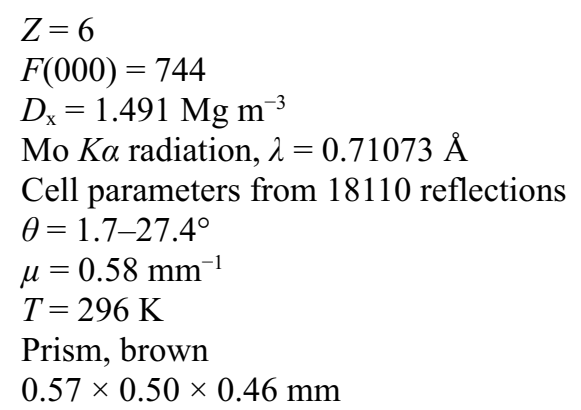

16045 measured reflections 6254 independent reflections 5000 reflections with $I>2 \sigma(I)$

$R_{\text {int }}=0.064$

$\theta_{\max }=26.0^{\circ}, \theta_{\min }=1.7^{\circ}$

$h=-8 \rightarrow 8$

$k=-14 \rightarrow 14$

$l=-23 \rightarrow 23$

Hydrogen site location: difference Fourier map

All $\mathrm{H}$-atom parameters refined

$w=1 /\left[\sigma^{2}\left(F_{\mathrm{o}}^{2}\right)+(0.056 P)^{2}\right]$

where $P=\left(F_{\mathrm{o}}^{2}+2 F_{\mathrm{c}}^{2}\right) / 3$

$(\Delta / \sigma)_{\max }=0.001$

$\Delta \rho_{\max }=0.34$ e $\AA^{-3}$

$\Delta \rho_{\min }=-0.28$ e $\AA^{-3}$ 
Extinction correction: SHELXL-2017/1

(Sheldrick 2015),

$\mathrm{Fc}^{*}=\mathrm{kFc}\left[1+0.001 \mathrm{xFc}^{2} \lambda^{3} / \sin (2 \theta)\right]^{-1 / 4}$

Extinction coefficient: 0.0327 (17)

\section{Special details}

Geometry. All esds (except the esd in the dihedral angle between two 1.s. planes) are estimated using the full covariance matrix. The cell esds are taken into account individually in the estimation of esds in distances, angles and torsion angles; correlations between esds in cell parameters are only used when they are defined by crystal symmetry. An approximate (isotropic) treatment of cell esds is used for estimating esds involving l.s. planes.

Fractional atomic coordinates and isotropic or equivalent isotropic displacement parameters $\left(\AA^{2}\right)$

\begin{tabular}{|c|c|c|c|c|}
\hline & $x$ & $y$ & $z$ & $U_{\text {iso }} * / U_{\text {eq }}$ \\
\hline $\mathrm{C} 11$ & $1.44783(7)$ & $0.25544(4)$ & $0.93231(2)$ & $0.04012(12)$ \\
\hline $\mathrm{Cl} 4$ & $0.55220(7)$ & $0.93572(4)$ & $0.73215(2)$ & $0.03912(12)$ \\
\hline $\mathrm{C} 15$ & $0.44034(7)$ & $0.39635(4)$ & $0.59956(2)$ & $0.04089(13)$ \\
\hline $\mathrm{Cl} 3$ & $0.85067(7)$ & $0.04098(4)$ & $0.91238(3)$ & $0.04840(14)$ \\
\hline $\mathrm{Cl} 6$ & $-0.13546(8)$ & $0.60301(5)$ & $0.58937(3)$ & $0.05432(15)$ \\
\hline $\mathrm{Cl} 2$ & $1.13465(8)$ & $0.15568(5)$ & $0.74387(3)$ & $0.05337(15)$ \\
\hline $\mathrm{O} 2$ & $0.9557(2)$ & $0.29533(13)$ & $0.93050(9)$ & $0.0492(3)$ \\
\hline $\mathrm{O} 1$ & $1.0439(2)$ & $-0.10288(14)$ & $0.73035(9)$ & $0.0517(4)$ \\
\hline N4 & $0.7622(2)$ & $0.41261(12)$ & $0.84094(8)$ & $0.0332(3)$ \\
\hline $\mathrm{O} 3$ & $0.0525(2)$ & $0.64579(14)$ & $0.39493(9)$ & $0.0553(4)$ \\
\hline N7 & $0.2335(2)$ & $0.81071(13)$ & $0.48888(8)$ & $0.0333(3)$ \\
\hline N5 & $0.6592(2)$ & $0.46067(13)$ & $0.74372(9)$ & $0.0347(3)$ \\
\hline N1 & $1.2273(2)$ & $-0.21584(13)$ & $0.82407(8)$ & $0.0334(3)$ \\
\hline N2 & $1.3362(2)$ & $-0.25722(14)$ & $0.92226(9)$ & $0.0376(4)$ \\
\hline N8 & $0.3400(2)$ & $0.90994(13)$ & $0.58704(9)$ & $0.0358(3)$ \\
\hline N6 & $0.7067(3)$ & $0.17781(15)$ & $0.79023(10)$ & $0.0427(4)$ \\
\hline N9 & $0.2868(3)$ & $0.60170(15)$ & $0.53791(10)$ & $0.0447(4)$ \\
\hline N3 & $1.2803(3)$ & $0.02017(15)$ & $0.86850(10)$ & $0.0421(4)$ \\
\hline $\mathrm{C} 14$ & $0.7037(2)$ & $0.55975(14)$ & $0.78881(9)$ & $0.0314(4)$ \\
\hline $\mathrm{C} 22$ & $0.2942(2)$ & $0.98527(15)$ & $0.54300(9)$ & $0.0323(4)$ \\
\hline $\mathrm{C} 9$ & $0.7708(2)$ & $0.52904(14)$ & $0.85148(9)$ & $0.0315(4)$ \\
\hline $\mathrm{C} 15$ & $0.6935(2)$ & $0.37525(15)$ & $0.77671(9)$ & $0.0335(4)$ \\
\hline $\mathrm{C} 1$ & $1.2214(2)$ & $-0.33227(14)$ & $0.81587(9)$ & $0.0324(4)$ \\
\hline $\mathrm{C} 17$ & $0.2255(2)$ & $0.92225(14)$ & $0.48021(9)$ & $0.0319(4)$ \\
\hline $\mathrm{C} 10$ & $0.8268(3)$ & $0.60738(16)$ & $0.90934(10)$ & $0.0369(4)$ \\
\hline $\mathrm{C} 18$ & $0.1652(2)$ & $0.97150(16)$ & $0.42385(10)$ & $0.0355(4)$ \\
\hline $\mathrm{C} 7$ & $1.2981(2)$ & $-0.17423(15)$ & $0.88773(9)$ & $0.0339(4)$ \\
\hline $\mathrm{C} 2$ & $1.1642(3)$ & $-0.41361(16)$ & $0.75996(10)$ & $0.0377(4)$ \\
\hline C6 & $1.2930(2)$ & $-0.35839(15)$ & $0.87927(10)$ & $0.0349(4)$ \\
\hline $\mathrm{C} 13$ & $0.6926(3)$ & $0.67112(16)$ & $0.78093(11)$ & $0.0385(4)$ \\
\hline $\mathrm{C} 23$ & $0.3047(2)$ & $0.80652(15)$ & $0.55283(9)$ & $0.0342(4)$ \\
\hline $\mathrm{C} 20$ & $0.2469(3)$ & $1.15029(16)$ & $0.49646(11)$ & $0.0392(4)$ \\
\hline $\mathrm{C} 21$ & $0.3055(3)$ & $1.10154(16)$ & $0.55279(11)$ & $0.0382(4)$ \\
\hline $\mathrm{C} 12$ & $0.7490(3)$ & $0.74911(16)$ & $0.83844(11)$ & $0.0394(4)$ \\
\hline $\mathrm{C} 5$ & $1.3092(3)$ & $-0.46915(17)$ & $0.88878(11)$ & $0.0421(4)$ \\
\hline
\end{tabular}




\begin{tabular}{|c|c|c|c|c|}
\hline $\mathrm{C} 19$ & $0.1785(3)$ & $1.08684(16)$ & $0.43313(10)$ & $0.0389(4)$ \\
\hline $\mathrm{C} 4$ & $1.2532(3)$ & $-0.54982(17)$ & $0.83351(12)$ & $0.0443(5)$ \\
\hline $\mathrm{C} 11$ & $0.8145(3)$ & $0.71817(16)$ & $0.90146(11)$ & $0.0401(4)$ \\
\hline $\mathrm{C} 8$ & $1.3424(3)$ & $-0.05475(17)$ & $0.91637(11)$ & $0.0414(4)$ \\
\hline $\mathrm{C} 16$ & $0.6469(3)$ & $0.25681(17)$ & $0.74466(11)$ & $0.0428(4)$ \\
\hline $\mathrm{C} 3$ & $1.1819(3)$ & $-0.52313(17)$ & $0.77020(12)$ & $0.0423(4)$ \\
\hline $\mathrm{C} 24$ & $0.3525(3)$ & $0.70519(18)$ & $0.58323(12)$ & $0.0458(5)$ \\
\hline $\mathrm{H} 2$ & $1.384(3)$ & $-0.2526(17)$ & $0.9558(11)$ & $0.028(5)^{*}$ \\
\hline H18 & $0.111(3)$ & $0.9325(18)$ & $0.3840(12)$ & $0.044(6)^{*}$ \\
\hline H10 & $0.873(3)$ & $0.5879(16)$ & $0.9522(11)$ & $0.033(5)^{*}$ \\
\hline $\mathrm{H} 2 \mathrm{~A}$ & $1.108(3)$ & $-0.3947(17)$ & $0.7161(11)$ & $0.039(5)^{*}$ \\
\hline H11 & $0.854(3)$ & $0.7742(19)$ & $0.9433(11)$ & $0.046(6)^{*}$ \\
\hline H19 & $0.136(3)$ & $1.1218(17)$ & $0.3927(11)$ & $0.039(5)^{*}$ \\
\hline $\mathrm{H} 20$ & $0.255(3)$ & $1.223(2)$ & $0.5012(12)$ & $0.048(6)^{*}$ \\
\hline H13 & $0.649(3)$ & $0.6891(17)$ & $0.7361(11)$ & $0.039(5)^{*}$ \\
\hline H12 & $0.740(3)$ & $0.816(2)$ & $0.8342(12)$ & $0.050(6)^{*}$ \\
\hline $\mathrm{H} 21$ & $0.350(3)$ & $1.1439(19)$ & $0.5987(12)$ & $0.047(6)^{*}$ \\
\hline H5 & $1.355(3)$ & $-0.4842(19)$ & $0.9333(12)$ & $0.050(6)^{*}$ \\
\hline H4 & $1.264(3)$ & $-0.6238(19)$ & $0.8385(11)$ & $0.040(5)^{*}$ \\
\hline H5A & $0.608(3)$ & $0.4556(18)$ & $0.7041(12)$ & $0.041(6)^{*}$ \\
\hline H1 & $1.179(3)$ & $-0.179(2)$ & $0.7956(13)$ & $0.055(7)^{*}$ \\
\hline $\mathrm{H} 4 \mathrm{~A}$ & $0.811(3)$ & $0.372(2)$ & $0.8683(12)$ & $0.052(6)^{*}$ \\
\hline H8 & $0.393(3)$ & $0.9236(17)$ & $0.6251(12)$ & $0.036(5)^{*}$ \\
\hline $\mathrm{H} 24 \mathrm{~A}$ & $0.291(3)$ & $0.710(2)$ & $0.6289(13)$ & $0.058(7)^{*}$ \\
\hline $\mathrm{H} 24 \mathrm{~B}$ & $0.491(5)$ & $0.697(3)$ & $0.5884(17)$ & $0.101(10)^{*}$ \\
\hline H7 & $0.183(4)$ & $0.757(2)$ & $0.4601(14)$ & $0.063(7)^{*}$ \\
\hline H9A & $0.167(4)$ & $0.600(2)$ & $0.5354(13)$ & $0.058(7)^{*}$ \\
\hline H16A & $0.518(4)$ & $0.253(2)$ & $0.7334(15)$ & $0.076(8)^{*}$ \\
\hline H9B & $0.325(4)$ & $0.551(2)$ & $0.5559(13)$ & $0.055(7)^{*}$ \\
\hline H9C & $0.336(4)$ & $0.593(2)$ & $0.4891(16)$ & $0.080(9)^{*}$ \\
\hline H16B & $0.709(3)$ & $0.2385(19)$ & $0.7019(13)$ & $0.052(6)^{*}$ \\
\hline $\mathrm{H} 3 \mathrm{C}$ & $1.143(3)$ & $-0.581(2)$ & $0.7340(12)$ & $0.050(6)^{*}$ \\
\hline H8A & $1.472(4)$ & $-0.045(2)$ & $0.9269(13)$ & $0.061(7)^{*}$ \\
\hline H8B & $1.282(4)$ & $-0.035(2)$ & $0.9596(14)$ & $0.066(7)^{*}$ \\
\hline H6A & $0.831(4)$ & $0.176(2)$ & $0.7947(13)$ & $0.061(8)^{*}$ \\
\hline H6B & $0.660(3)$ & $0.194(2)$ & $0.8388(14)$ & $0.059(7)^{*}$ \\
\hline $\mathrm{H} 6 \mathrm{C}$ & $0.667(4)$ & $0.118(3)$ & $0.7738(15)$ & $0.065(8)^{*}$ \\
\hline $\mathrm{H} 2 \mathrm{C}$ & $0.920(3)$ & $0.2322(16)$ & $0.9337(14)$ & $0.063(8)^{*}$ \\
\hline $\mathrm{H} 2 \mathrm{D}$ & $1.072(2)$ & $0.289(2)$ & $0.9267(17)$ & $0.098(12)^{*}$ \\
\hline $\mathrm{H} 3 \mathrm{~A}$ & $1.316(4)$ & $0.087(3)$ & $0.8871(16)$ & $0.086(10)^{*}$ \\
\hline H3B & $1.330(3)$ & $0.000(2)$ & $0.8197(14)$ & $0.060(7)^{*}$ \\
\hline H3 & $1.154(4)$ & $0.022(2)$ & $0.8652(12)$ & $0.056(7)^{*}$ \\
\hline $\mathrm{H} 3 \mathrm{D}$ & $-0.062(3)$ & $0.649(3)$ & $0.3941(19)$ & $0.106(12)^{*}$ \\
\hline $\mathrm{H} 3 \mathrm{E}$ & $0.082(4)$ & $0.5804(16)$ & $0.3962(15)$ & $0.071(9)^{*}$ \\
\hline H1A & $0.926(3)$ & $-0.102(3)$ & $0.7339(18)$ & $0.100(12)^{*}$ \\
\hline $\mathrm{H} 2 \mathrm{~B}$ & $1.076(4)$ & $-0.0391(16)$ & $0.7286(15)$ & $0.069(9)^{*}$ \\
\hline
\end{tabular}


Atomic displacement parameters $\left(\AA^{2}\right)$

\begin{tabular}{|c|c|c|c|c|c|c|}
\hline & $U^{11}$ & $U^{22}$ & $U^{33}$ & $U^{12}$ & $U^{13}$ & $U^{23}$ \\
\hline $\mathrm{Cl1}$ & $0.0439(2)$ & $0.0399(2)$ & $0.0360(2)$ & 0.00037 (18) & $-0.00373(18)$ & $0.00530(18)$ \\
\hline $\mathrm{Cl} 4$ & $0.0430(2)$ & $0.0388(2)$ & $0.0348(2)$ & $-0.00156(18)$ & $-0.00346(18)$ & $0.00436(18)$ \\
\hline $\mathrm{Cl} 5$ & $0.0447(3)$ & $0.0395(2)$ & $0.0378(2)$ & $-0.00330(18)$ & $-0.00542(18)$ & $0.00547(18)$ \\
\hline $\mathrm{Cl} 3$ & 0.0486 & 0.0458 & 0.0518 & $-0.0026(2)$ & $0.0038(2)$ & $0.0112(2)$ \\
\hline $\mathrm{Cl} 6$ & 0.0503 & 0.0508 & 0.0615 & $0.0005(2)$ & $-0.0013(2)$ & $0.0085(2)$ \\
\hline $\mathrm{Cl} 2$ & 0.0491 & 0.0519 & $0.0600(3)$ & $-0.0049(2)$ & $-0.0007(2)$ & $0.0126(2)$ \\
\hline $\mathrm{O} 2$ & $0.0490(9)$ & $0.0458(9)$ & $0.0538(9)$ & $-0.0016(7)$ & $-0.0076(7)$ & $0.0121(7)$ \\
\hline $\mathrm{O} 1$ & $0.0475(9)$ & $0.0546(9)$ & $0.0566(9)$ & $-0.0045(7)$ & $-0.0098(7)$ & $0.0209(8)$ \\
\hline N4 & $0.0346(7)$ & $0.0320(7)$ & $0.0337(8)$ & $0.0003(6)$ & $-0.0023(6)$ & $0.0071(6)$ \\
\hline $\mathrm{O} 3$ & $0.0496(9)$ & $0.0456(9)$ & $0.0652(10)$ & $0.0011(7)$ & $-0.0110(8)$ & $-0.0056(7)$ \\
\hline N7 & $0.0323(7)$ & $0.0331(8)$ & $0.0331(8)$ & $-0.0025(6)$ & $-0.0008(6)$ & $0.0017(6)$ \\
\hline N5 & $0.0336(8)$ & $0.0387(8)$ & $0.0314(8)$ & $0.0013(6)$ & $-0.0032(6)$ & $0.0047(6)$ \\
\hline N1 & $0.0317(7)$ & $0.0359(8)$ & $0.0341(8)$ & $-0.0008(6)$ & $-0.0022(6)$ & $0.0106(6)$ \\
\hline N2 & $0.0333(8)$ & $0.0494(10)$ & $0.0314(9)$ & $-0.0017(7)$ & $-0.0053(7)$ & $0.0107(7)$ \\
\hline N8 & $0.0342(8)$ & $0.0420(9)$ & $0.0298(8)$ & $-0.0059(6)$ & $-0.0038(6)$ & $0.0028(6)$ \\
\hline N6 & $0.0505(11)$ & $0.0310(9)$ & $0.0451(10)$ & $-0.0057(8)$ & $0.0012(8)$ & $0.0025(7)$ \\
\hline N9 & $0.0501(11)$ & $0.0375(9)$ & $0.0485(11)$ & $0.0051(8)$ & $0.0010(8)$ & $0.0128(8)$ \\
\hline N3 & $0.0469(10)$ & $0.0358(9)$ & $0.0426(10)$ & $-0.0042(7)$ & $0.0018(8)$ & $0.0034(7)$ \\
\hline $\mathrm{C} 14$ & $0.0264(8)$ & $0.0346(9)$ & $0.0331(9)$ & $0.0007(6)$ & $0.0018(7)$ & $0.0052(7)$ \\
\hline $\mathrm{C} 22$ & $0.0265(8)$ & $0.0378(9)$ & $0.0315(9)$ & $-0.0035(7)$ & $0.0011(7)$ & $0.0027(7)$ \\
\hline $\mathrm{C} 9$ & $0.0275(8)$ & $0.0312(8)$ & $0.0357(9)$ & $-0.0003(6)$ & $0.0023(7)$ & $0.0054(7)$ \\
\hline $\mathrm{C} 15$ & $0.0295(8)$ & $0.0347(9)$ & $0.0353(9)$ & $0.0001(7)$ & $0.0003(7)$ & $0.0029(7)$ \\
\hline $\mathrm{C} 1$ & $0.0266(8)$ & $0.0355(9)$ & $0.0361(9)$ & $-0.0010(7)$ & $0.0026(7)$ & $0.0093(7)$ \\
\hline $\mathrm{C} 17$ & $0.0274(8)$ & $0.0337(9)$ & $0.0334(9)$ & $-0.0023(6)$ & $0.0034(7)$ & $0.0019(7)$ \\
\hline $\mathrm{C} 10$ & $0.0349(9)$ & $0.0406(10)$ & $0.0343(9)$ & $-0.0015(7)$ & $0.0003(7)$ & $0.0042(8)$ \\
\hline C18 & $0.0306(8)$ & $0.0423(10)$ & $0.0320(9)$ & $-0.0003(7)$ & $0.0002(7)$ & $0.0016(8)$ \\
\hline $\mathrm{C} 7$ & $0.0287(8)$ & $0.0391(9)$ & $0.0343(9)$ & $-0.0018(7)$ & $0.0006(7)$ & $0.0073(7)$ \\
\hline $\mathrm{C} 2$ & $0.0319(9)$ & $0.0440(10)$ & $0.0371(10)$ & $-0.0025(7)$ & $0.0017(7)$ & $0.0066(8)$ \\
\hline C6 & $0.0269(8)$ & $0.0417(10)$ & $0.0378(9)$ & $0.0014(7)$ & $0.0008(7)$ & $0.0112(8)$ \\
\hline C13 & $0.0334(9)$ & $0.0399(10)$ & $0.0442(11)$ & $0.0032(7)$ & $0.0036(8)$ & $0.0130(8)$ \\
\hline $\mathrm{C} 23$ & $0.0291(8)$ & $0.0384(9)$ & $0.0353(9)$ & $-0.0026(7)$ & $0.0008(7)$ & $0.0066(7)$ \\
\hline $\mathrm{C} 20$ & $0.0344(9)$ & $0.0343(10)$ & $0.0485(11)$ & $-0.0028(8)$ & $0.0075(8)$ & $0.0051(8)$ \\
\hline $\mathrm{C} 21$ & $0.0318(9)$ & $0.0384(10)$ & $0.0408(10)$ & $-0.0061(7)$ & $0.0020(8)$ & $-0.0030(8)$ \\
\hline $\mathrm{C} 12$ & $0.0359(9)$ & $0.0307(9)$ & $0.0519(12)$ & $0.0024(7)$ & $0.0068(8)$ & $0.0076(8)$ \\
\hline C5 & $0.0339(9)$ & 0.0470 & $0.0498(12)$ & $0.0041(8)$ & $0.0033(8)$ & $0.0202(9)$ \\
\hline C19 & $0.0349(9)$ & $0.0419(10)$ & $0.0412(10)$ & $0.0012(7)$ & $0.0053(8)$ & $0.0100(8)$ \\
\hline $\mathrm{C} 4$ & $0.0354(10)$ & $0.0389(10)$ & $0.0608(13)$ & $0.0044(8)$ & $0.0106(9)$ & $0.0146(10)$ \\
\hline C11 & $0.0351(9)$ & $0.0367(10)$ & $0.0457(11)$ & $-0.0036(7)$ & $0.0058(8)$ & $-0.0013(8)$ \\
\hline $\mathrm{C} 8$ & 0.0423 & $0.0437(11)$ & $0.0362(10)$ & $-0.0059(8)$ & $-0.0033(9)$ & $0.0017(8)$ \\
\hline $\mathrm{C} 16$ & $0.0494(12)$ & $0.0369(10)$ & $0.0398(11)$ & $-0.0013(8)$ & $-0.0073(9)$ & $-0.0001(8)$ \\
\hline $\mathrm{C} 3$ & $0.0349(9)$ & $0.0401(10)$ & $0.0509(12)$ & $-0.0025(8)$ & $0.0069(8)$ & $0.0044(9)$ \\
\hline $\mathrm{C} 24$ & $0.0478(11)$ & $0.0460(11)$ & $0.0456(12)$ & $-0.0031(9)$ & $-0.0062(9)$ & $0.0141(9)$ \\
\hline
\end{tabular}


Geometric parameters $\left(\AA,{ }^{\circ}\right)$

\begin{tabular}{|c|c|c|c|}
\hline $\mathrm{O} 2-\mathrm{H} 2 \mathrm{C}$ & $0.817(16)$ & $\mathrm{C} 22-\mathrm{C} 17$ & $1.391(2)$ \\
\hline $\mathrm{O} 2-\mathrm{H} 2 \mathrm{D}$ & $0.812(17)$ & $\mathrm{C} 22-\mathrm{C} 21$ & $1.391(3)$ \\
\hline $\mathrm{O} 1-\mathrm{H} 1 \mathrm{~A}$ & $0.819(17)$ & $\mathrm{C} 9-\mathrm{C} 10$ & $1.383(3)$ \\
\hline $\mathrm{O} 1-\mathrm{H} 2 \mathrm{~B}$ & $0.810(16)$ & $\mathrm{C} 15-\mathrm{C} 16$ & $1.494(3)$ \\
\hline $\mathrm{N} 1-\mathrm{C} 7$ & $1.328(2)$ & $\mathrm{C} 1-\mathrm{C} 2$ & $1.381(3)$ \\
\hline $\mathrm{N} 1-\mathrm{C} 1$ & $1.393(2)$ & $\mathrm{C} 1-\mathrm{C} 6$ & $1.396(2)$ \\
\hline $\mathrm{N} 1-\mathrm{H} 1$ & $0.83(2)$ & $\mathrm{C} 17-\mathrm{C} 18$ & $1.384(2)$ \\
\hline $\mathrm{N} 2-\mathrm{C} 7$ & $1.322(2)$ & $\mathrm{C} 10-\mathrm{C} 11$ & $1.380(3)$ \\
\hline $\mathrm{N} 2-\mathrm{C} 6$ & $1.386(3)$ & $\mathrm{C} 10-\mathrm{H} 10$ & $0.95(2)$ \\
\hline $\mathrm{N} 2-\mathrm{H} 2$ & $0.71(2)$ & $\mathrm{C} 18-\mathrm{C} 19$ & $1.381(3)$ \\
\hline $\mathrm{N} 3-\mathrm{C} 8$ & $1.461(3)$ & C18-H18 & $0.91(2)$ \\
\hline $\mathrm{N} 3-\mathrm{H} 3 \mathrm{~A}$ & $0.87(4)$ & $\mathrm{C} 7-\mathrm{C} 8$ & $1.488(3)$ \\
\hline N3-H3B & $0.99(3)$ & $\mathrm{C} 2-\mathrm{C} 3$ & $1.381(3)$ \\
\hline N3-H3 & $0.88(3)$ & $\mathrm{C} 2-\mathrm{H} 2 \mathrm{~A}$ & $0.99(2)$ \\
\hline $\mathrm{N} 4-\mathrm{C} 15$ & $1.322(2)$ & $\mathrm{C} 6-\mathrm{C} 5$ & $1.390(3)$ \\
\hline $\mathrm{N} 4-\mathrm{C} 9$ & $1.392(2)$ & $\mathrm{C} 13-\mathrm{C} 12$ & $1.377(3)$ \\
\hline $\mathrm{N} 4-\mathrm{H} 4 \mathrm{~A}$ & $0.85(2)$ & $\mathrm{C} 13-\mathrm{H} 13$ & $0.97(2)$ \\
\hline N5-C15 & $1.324(2)$ & $\mathrm{C} 23-\mathrm{C} 24$ & $1.484(3)$ \\
\hline N5-C14 & $1.386(2)$ & $\mathrm{C} 20-\mathrm{C} 21$ & $1.377(3)$ \\
\hline N5-H5A & $0.83(2)$ & $\mathrm{C} 20-\mathrm{C} 19$ & $1.400(3)$ \\
\hline N6-C16 & $1.462(3)$ & $\mathrm{C} 20-\mathrm{H} 20$ & $0.87(2)$ \\
\hline N6-H6A & $0.86(3)$ & $\mathrm{C} 21-\mathrm{H} 21$ & $0.99(2)$ \\
\hline N6-H6B & $0.98(3)$ & $\mathrm{C} 12-\mathrm{C} 11$ & $1.399(3)$ \\
\hline N6- $\mathrm{H} 6 \mathrm{C}$ & $0.79(3)$ & $\mathrm{C} 12-\mathrm{H} 12$ & $0.83(2)$ \\
\hline $\mathrm{O} 3-\mathrm{H} 3 \mathrm{D}$ & $0.796(17)$ & $\mathrm{C} 5-\mathrm{C} 4$ & $1.366(3)$ \\
\hline $\mathrm{O} 3-\mathrm{H} 3 \mathrm{E}$ & $0.822(16)$ & $\mathrm{C} 5-\mathrm{H} 5$ & $0.96(2)$ \\
\hline $\mathrm{N} 7-\mathrm{C} 23$ & $1.328(2)$ & C19-H19 & $0.99(2)$ \\
\hline $\mathrm{N} 7-\mathrm{C} 17$ & $1.391(2)$ & $\mathrm{C} 4-\mathrm{C} 3$ & $1.397(3)$ \\
\hline $\mathrm{N} 7-\mathrm{H} 7$ & $0.85(3)$ & $\mathrm{C} 4-\mathrm{H} 4$ & $0.92(2)$ \\
\hline $\mathrm{N} 8-\mathrm{C} 23$ & $1.332(2)$ & C11-H11 & $0.99(2)$ \\
\hline $\mathrm{N} 8-\mathrm{C} 22$ & $1.383(2)$ & $\mathrm{C} 8-\mathrm{H} 8 \mathrm{~A}$ & $0.92(3)$ \\
\hline N8- $\mathrm{H} 8$ & $0.80(2)$ & $\mathrm{C} 8-\mathrm{H} 8 \mathrm{~B}$ & $0.93(3)$ \\
\hline N9-C24 & $1.467(3)$ & $\mathrm{C} 16-\mathrm{H} 16 \mathrm{~A}$ & $0.92(3)$ \\
\hline N9-H9A & $0.83(3)$ & $\mathrm{C} 16-\mathrm{H} 16 \mathrm{~B}$ & $0.93(2)$ \\
\hline N9-H9B & $0.80(3)$ & $\mathrm{C} 3-\mathrm{H} 3 \mathrm{C}$ & $0.93(2)$ \\
\hline N9- $\mathrm{H} 9 \mathrm{C}$ & $0.99(3)$ & $\mathrm{C} 24-\mathrm{H} 24 \mathrm{~A}$ & $0.97(2)$ \\
\hline $\mathrm{C} 14-\mathrm{C} 13$ & $1.386(2)$ & $\mathrm{C} 24-\mathrm{H} 24 \mathrm{~B}$ & $0.97(3)$ \\
\hline $\mathrm{C} 14-\mathrm{C} 9$ & $1.395(2)$ & & \\
\hline $\mathrm{H} 2 \mathrm{C}-\mathrm{O} 2-\mathrm{H} 2 \mathrm{D}$ & $104(2)$ & $\mathrm{C} 19-\mathrm{C} 18-\mathrm{C} 17$ & $116.33(18)$ \\
\hline $\mathrm{H} 1 \mathrm{~A}-\mathrm{O} 1-\mathrm{H} 2 \mathrm{~B}$ & $106(2)$ & $\mathrm{C} 19-\mathrm{C} 18-\mathrm{H} 18$ & $120.3(14)$ \\
\hline $\mathrm{C} 15-\mathrm{N} 4-\mathrm{C} 9$ & $108.99(15)$ & $\mathrm{C} 17-\mathrm{C} 18-\mathrm{H} 18$ & $123.2(14)$ \\
\hline $\mathrm{C} 15-\mathrm{N} 4-\mathrm{H} 4 \mathrm{~A}$ & $124.8(16)$ & $\mathrm{N} 2-\mathrm{C} 7-\mathrm{N} 1$ & $109.33(16)$ \\
\hline $\mathrm{C} 9-\mathrm{N} 4-\mathrm{H} 4 \mathrm{~A}$ & $125.4(16)$ & $\mathrm{N} 2-\mathrm{C} 7-\mathrm{C} 8$ & $123.45(17)$ \\
\hline $\mathrm{H} 3 \mathrm{D}-\mathrm{O} 3-\mathrm{H} 3 \mathrm{E}$ & $107(2)$ & $\mathrm{N} 1-\mathrm{C} 7-\mathrm{C} 8$ & $127.11(16)$ \\
\hline $\mathrm{C} 23-\mathrm{N} 7-\mathrm{C} 17$ & $108.55(16)$ & $\mathrm{C} 3-\mathrm{C} 2-\mathrm{C} 1$ & $116.08(18)$ \\
\hline
\end{tabular}




\begin{tabular}{|c|c|c|c|}
\hline $\mathrm{C} 23-\mathrm{N} 7-\mathrm{H} 7$ & $126.6(17)$ & $\mathrm{C} 3-\mathrm{C} 2-\mathrm{H} 2 \mathrm{~A}$ & $121.8(12)$ \\
\hline $\mathrm{C} 17-\mathrm{N} 7-\mathrm{H} 7$ & $124.0(17)$ & $\mathrm{C} 1-\mathrm{C} 2-\mathrm{H} 2 \mathrm{~A}$ & $122.0(12)$ \\
\hline $\mathrm{C} 15-\mathrm{N} 5-\mathrm{C} 14$ & $109.14(15)$ & $\mathrm{N} 2-\mathrm{C} 6-\mathrm{C} 5$ & $132.85(18)$ \\
\hline $\mathrm{C} 15-\mathrm{N} 5-\mathrm{H} 5 \mathrm{~A}$ & $124.9(15)$ & $\mathrm{N} 2-\mathrm{C} 6-\mathrm{C} 1$ & $106.35(15)$ \\
\hline $\mathrm{C} 14-\mathrm{N} 5-\mathrm{H} 5 \mathrm{~A}$ & $125.6(15)$ & $\mathrm{C} 5-\mathrm{C} 6-\mathrm{C} 1$ & $120.80(18)$ \\
\hline $\mathrm{C} 7-\mathrm{N} 1-\mathrm{C} 1$ & $109.27(15)$ & $\mathrm{C} 12-\mathrm{C} 13-\mathrm{C} 14$ & $116.35(17)$ \\
\hline $\mathrm{C} 7-\mathrm{N} 1-\mathrm{H} 1$ & $125.7(17)$ & $\mathrm{C} 12-\mathrm{C} 13-\mathrm{H} 13$ & $124.6(12)$ \\
\hline $\mathrm{C} 1-\mathrm{N} 1-\mathrm{H} 1$ & $124.5(17)$ & $\mathrm{C} 14-\mathrm{C} 13-\mathrm{H} 13$ & $119.1(12)$ \\
\hline $\mathrm{C} 7-\mathrm{N} 2-\mathrm{C} 6$ & $109.38(16)$ & $\mathrm{N} 7-\mathrm{C} 23-\mathrm{N} 8$ & $109.72(16)$ \\
\hline $\mathrm{C} 7-\mathrm{N} 2-\mathrm{H} 2$ & $126.4(17)$ & $\mathrm{N} 7-\mathrm{C} 23-\mathrm{C} 24$ & $127.55(18)$ \\
\hline $\mathrm{C} 6-\mathrm{N} 2-\mathrm{H} 2$ & $123.7(17)$ & $\mathrm{N} 8-\mathrm{C} 23-\mathrm{C} 24$ & $122.63(17)$ \\
\hline $\mathrm{C} 23-\mathrm{N} 8-\mathrm{C} 22$ & $108.85(15)$ & $\mathrm{C} 21-\mathrm{C} 20-\mathrm{C} 19$ & 122.08 \\
\hline $\mathrm{C} 23-\mathrm{N} 8-\mathrm{H} 8$ & $123.7(15)$ & $\mathrm{C} 21-\mathrm{C} 20-\mathrm{H} 20$ & $117.6(16)$ \\
\hline $\mathrm{C} 22-\mathrm{N} 8-\mathrm{H} 8$ & $127.0(15)$ & $\mathrm{C} 19-\mathrm{C} 20-\mathrm{H} 20$ & $120.3(15)$ \\
\hline $\mathrm{C} 16-\mathrm{N} 6-\mathrm{H} 6 \mathrm{~A}$ & $111.4(17)$ & $\mathrm{C} 20-\mathrm{C} 21-\mathrm{C} 22$ & $116.12(18)$ \\
\hline $\mathrm{C} 16-\mathrm{N} 6-\mathrm{H} 6 \mathrm{~B}$ & $115.5(14)$ & $\mathrm{C} 20-\mathrm{C} 21-\mathrm{H} 21$ & $124.1(13)$ \\
\hline $\mathrm{H} 6 \mathrm{~A}-\mathrm{N} 6-\mathrm{H} 6 \mathrm{~B}$ & $104(2)$ & $\mathrm{C} 22-\mathrm{C} 21-\mathrm{H} 21$ & 119.7 (13) \\
\hline $\mathrm{C} 16-\mathrm{N} 6-\mathrm{H} 6 \mathrm{C}$ & $109(2)$ & $\mathrm{C} 13-\mathrm{C} 12-\mathrm{C} 11$ & $122.09(18)$ \\
\hline $\mathrm{H} 6 \mathrm{~A}-\mathrm{N} 6-\mathrm{H} 6 \mathrm{C}$ & $110(3)$ & $\mathrm{C} 13-\mathrm{C} 12-\mathrm{H} 12$ & $116.6(16)$ \\
\hline $\mathrm{H} 6 \mathrm{~B}-\mathrm{N} 6-\mathrm{H} 6 \mathrm{C}$ & $107(2)$ & $\mathrm{C} 11-\mathrm{C} 12-\mathrm{H} 12$ & $121.3(16)$ \\
\hline $\mathrm{C} 24-\mathrm{N} 9-\mathrm{H} 9 \mathrm{~A}$ & $110.4(18)$ & $\mathrm{C} 4-\mathrm{C} 5-\mathrm{C} 6$ & $116.97(18)$ \\
\hline $\mathrm{C} 24-\mathrm{N} 9-\mathrm{H} 9 \mathrm{~B}$ & $106.7(18)$ & $\mathrm{C} 4-\mathrm{C} 5-\mathrm{H} 5$ & $124.2(14)$ \\
\hline $\mathrm{H} 9 \mathrm{~A}-\mathrm{N} 9-\mathrm{H} 9 \mathrm{~B}$ & $109(2)$ & C6- $55-\mathrm{H} 5$ & $118.8(14)$ \\
\hline $\mathrm{C} 24-\mathrm{N} 9-\mathrm{H} 9 \mathrm{C}$ & $113.6(17)$ & $\mathrm{C} 18-\mathrm{C} 19-\mathrm{C} 20$ & $121.68(18)$ \\
\hline $\mathrm{H} 9 \mathrm{~A}-\mathrm{N} 9-\mathrm{H} 9 \mathrm{C}$ & $108(2)$ & $\mathrm{C} 18-\mathrm{C} 19-\mathrm{H} 19$ & $116.2(12)$ \\
\hline $\mathrm{H} 9 \mathrm{~B}-\mathrm{N} 9-\mathrm{H} 9 \mathrm{C}$ & $109(2)$ & $\mathrm{C} 20-\mathrm{C} 19-\mathrm{H} 19$ & $122.1(12)$ \\
\hline $\mathrm{C} 8-\mathrm{N} 3-\mathrm{H} 3 \mathrm{~A}$ & $108(2)$ & $\mathrm{C} 5-\mathrm{C} 4-\mathrm{C} 3$ & $121.94(19)$ \\
\hline $\mathrm{C} 8-\mathrm{N} 3-\mathrm{H} 3 \mathrm{~B}$ & $114.2(14)$ & $\mathrm{C} 5-\mathrm{C} 4-\mathrm{H} 4$ & $118.4(13)$ \\
\hline $\mathrm{H} 3 \mathrm{~A}-\mathrm{N} 3-\mathrm{H} 3 \mathrm{~B}$ & $111(2)$ & $\mathrm{C} 3-\mathrm{C} 4-\mathrm{H} 4$ & $119.6(13)$ \\
\hline $\mathrm{C} 8-\mathrm{N} 3-\mathrm{H} 3$ & $111.0(16)$ & $\mathrm{C} 10-\mathrm{C} 11-\mathrm{C} 12$ & $121.71(19)$ \\
\hline $\mathrm{H} 3 \mathrm{~A}-\mathrm{N} 3-\mathrm{H} 3$ & $106(3)$ & $\mathrm{C} 10-\mathrm{C} 11-\mathrm{H} 11$ & $115.9(12)$ \\
\hline $\mathrm{H} 3 \mathrm{~B}-\mathrm{N} 3-\mathrm{H} 3$ & $107(2)$ & $\mathrm{C} 12-\mathrm{C} 11-\mathrm{H} 11$ & $122.3(12)$ \\
\hline $\mathrm{C} 13-\mathrm{C} 14-\mathrm{N} 5$ & $132.44(17)$ & $\mathrm{N} 3-\mathrm{C} 8-\mathrm{C} 7$ & $112.34(16)$ \\
\hline $\mathrm{C} 13-\mathrm{C} 14-\mathrm{C} 9$ & $121.46(17)$ & $\mathrm{N} 3-\mathrm{C} 8-\mathrm{H} 8 \mathrm{~A}$ & $110.4(16)$ \\
\hline $\mathrm{N} 5-\mathrm{C} 14-\mathrm{C} 9$ & $106.11(15)$ & $\mathrm{C} 7-\mathrm{C} 8-\mathrm{H} 8 \mathrm{~A}$ & $110.8(16)$ \\
\hline $\mathrm{N} 8-\mathrm{C} 22-\mathrm{C} 17$ & $106.45(15)$ & $\mathrm{N} 3-\mathrm{C} 8-\mathrm{H} 8 \mathrm{~B}$ & $109.5(16)$ \\
\hline $\mathrm{N} 8-\mathrm{C} 22-\mathrm{C} 21$ & $131.74(17)$ & $\mathrm{C} 7-\mathrm{C} 8-\mathrm{H} 8 \mathrm{~B}$ & $109.3(16)$ \\
\hline $\mathrm{C} 17-\mathrm{C} 22-\mathrm{C} 21$ & $121.81(17)$ & $\mathrm{H} 8 \mathrm{~A}-\mathrm{C} 8-\mathrm{H} 8 \mathrm{~B}$ & $104(2)$ \\
\hline $\mathrm{C} 10-\mathrm{C} 9-\mathrm{N} 4$ & $131.77(16)$ & $\mathrm{N} 6-\mathrm{C} 16-\mathrm{C} 15$ & $112.13(17)$ \\
\hline $\mathrm{C} 10-\mathrm{C} 9-\mathrm{C} 14$ & $122.19(16)$ & $\mathrm{N} 6-\mathrm{C} 16-\mathrm{H} 16 \mathrm{~A}$ & $113.4(18)$ \\
\hline $\mathrm{N} 4-\mathrm{C} 9-\mathrm{C} 14$ & $106.02(15)$ & $\mathrm{C} 15-\mathrm{C} 16-\mathrm{H} 16 \mathrm{~A}$ & $108.1(18)$ \\
\hline $\mathrm{N} 4-\mathrm{C} 15-\mathrm{N} 5$ & $109.73(16)$ & $\mathrm{N} 6-\mathrm{C} 16-\mathrm{H} 16 \mathrm{~B}$ & $108.5(14)$ \\
\hline $\mathrm{N} 4-\mathrm{C} 15-\mathrm{C} 16$ & $127.44(16)$ & $\mathrm{C} 15-\mathrm{C} 16-\mathrm{H} 16 \mathrm{~B}$ & $109.5(15)$ \\
\hline $\mathrm{N} 5-\mathrm{C} 15-\mathrm{C} 16$ & $122.72(16)$ & $\mathrm{H} 16 \mathrm{~A}-\mathrm{C} 16-\mathrm{H} 16 \mathrm{~B}$ & $105(2)$ \\
\hline $\mathrm{C} 2-\mathrm{C} 1-\mathrm{N} 1$ & $131.96(16)$ & $\mathrm{C} 2-\mathrm{C} 3-\mathrm{C} 4$ & $121.8(2)$ \\
\hline $\mathrm{C} 2-\mathrm{C} 1-\mathrm{C} 6$ & $122.37(17)$ & $\mathrm{C} 2-\mathrm{C} 3-\mathrm{H} 3 \mathrm{C}$ & $118.8(14)$ \\
\hline $\mathrm{N} 1-\mathrm{C} 1-\mathrm{C} 6$ & $105.66(16)$ & $\mathrm{C} 4-\mathrm{C} 3-\mathrm{H} 3 \mathrm{C}$ & $119.4(14)$ \\
\hline $\mathrm{C} 18-\mathrm{C} 17-\mathrm{C} 22$ & $121.97(16)$ & $\mathrm{N} 9-\mathrm{C} 24-\mathrm{C} 23$ & $112.39(17)$ \\
\hline
\end{tabular}




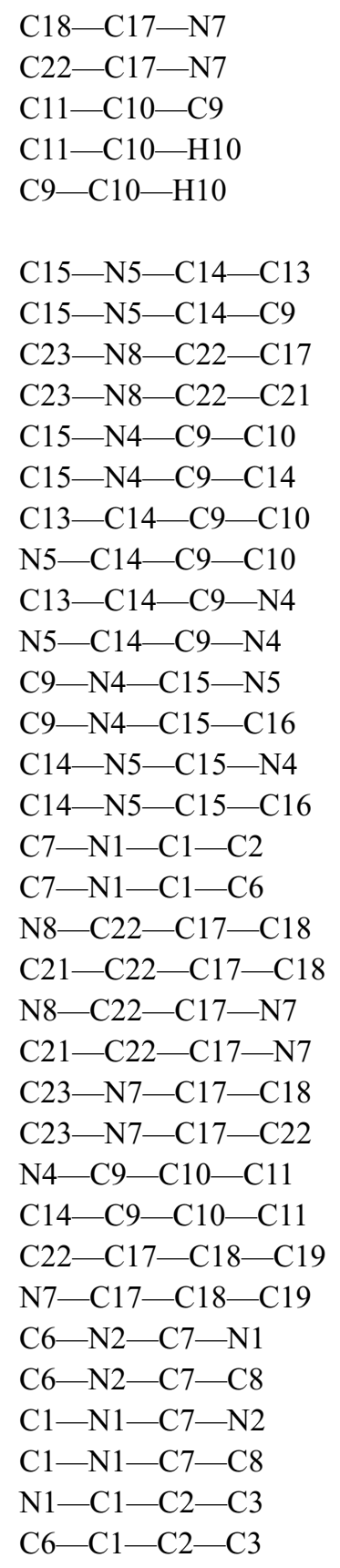

$131.61(17)$

$106.41(15)$

$116.19(17)$

$120.6(12)$

$123.2(12)$

$-179.30(18)$

$0.93(19)$

$1.03(19)$

$-179.49(17)$

$178.27(18)$

-0.41 (18)

$1.1(3)$

$-179.15(15)$

$179.89(15)$

$-0.31(18)$

$1.02(19)$

$-175.38(18)$

$-1.22(19)$

$175.38(16)$

$-178.68(18)$

$0.28(18)$

$179.76(15)$

$0.2(2)$

$-0.25(17)$

$-179.79(15)$

$179.37(18)$

$-0.62(18)$

-179.14 (17)

$-0.6(2)$

$0.5(2)$

$-179.44(17)$

$1.4(2)$

$-175.02(16)$

$-1.02(19)$

$175.20(17)$

178.75 (17)

$-0.1(2)$

$$
\begin{aligned}
& \text { N9-C24-H24A } \\
& \text { C23-C } 24-\mathrm{H} 24 \mathrm{~A} \\
& \text { N9-C24-H24B } \\
& \text { C23-C24-H24B } \\
& \text { H24A-C24-H24B }
\end{aligned}
$$

$\mathrm{C} 7-\mathrm{N} 2-\mathrm{C} 6-\mathrm{C} 5$

C7-N2-C6-C1

$\mathrm{C} 2-\mathrm{C} 1-\mathrm{C} 6-\mathrm{N} 2$

$\mathrm{N} 1-\mathrm{C} 1-\mathrm{C} 6-\mathrm{N} 2$

$\mathrm{C} 2-\mathrm{C} 1-\mathrm{C} 6-\mathrm{C} 5$

$\mathrm{N} 1-\mathrm{C} 1-\mathrm{C} 6-\mathrm{C} 5$

$\mathrm{N} 5-\mathrm{C} 14-\mathrm{C} 13-\mathrm{C} 12$

C9- $14-\mathrm{C} 13-\mathrm{C} 12$

$\mathrm{C} 17-\mathrm{N} 7-\mathrm{C} 23-\mathrm{N} 8$

$\mathrm{C} 17-\mathrm{N} 7-\mathrm{C} 23-\mathrm{C} 24$

$\mathrm{C} 22-\mathrm{N} 8-\mathrm{C} 23-\mathrm{N} 7$

$\mathrm{C} 22-\mathrm{N} 8-\mathrm{C} 23-\mathrm{C} 24$

$\mathrm{C} 19-\mathrm{C} 20-\mathrm{C} 21-\mathrm{C} 22$

$\mathrm{N} 8-\mathrm{C} 22-\mathrm{C} 21-\mathrm{C} 20$

$\mathrm{C} 17-\mathrm{C} 22-\mathrm{C} 21-\mathrm{C} 20$

$\mathrm{C} 14-\mathrm{C} 13-\mathrm{C} 12-\mathrm{C} 11$

$\mathrm{N} 2-\mathrm{C} 6-\mathrm{C} 5-\mathrm{C} 4$

$\mathrm{C} 1-\mathrm{C} 6-\mathrm{C} 5-\mathrm{C} 4$

$\mathrm{C} 17-\mathrm{C} 18-\mathrm{C} 19-\mathrm{C} 20$

$\mathrm{C} 21-\mathrm{C} 20-\mathrm{C} 19-\mathrm{C} 18$

$\mathrm{C} 6-\mathrm{C} 5-\mathrm{C} 4-\mathrm{C} 3$

$\mathrm{C} 9-\mathrm{C} 10-\mathrm{C} 11-\mathrm{C} 12$

$\mathrm{C} 13-\mathrm{C} 12-\mathrm{C} 11-\mathrm{C} 10$

$\mathrm{N} 2-\mathrm{C} 7-\mathrm{C} 8-\mathrm{N} 3$

$\mathrm{N} 1-\mathrm{C} 7-\mathrm{C} 8-\mathrm{N} 3$

$\mathrm{N} 4-\mathrm{C} 15-\mathrm{C} 16-\mathrm{N} 6$

$\mathrm{N} 5-\mathrm{C} 15-\mathrm{C} 16-\mathrm{N} 6$

$\mathrm{C} 1-\mathrm{C} 2-\mathrm{C} 3-\mathrm{C} 4$

$\mathrm{C} 5-\mathrm{C} 4-\mathrm{C} 3-\mathrm{C} 2$

$\mathrm{N} 7-\mathrm{C} 23-\mathrm{C} 24-\mathrm{N} 9$

$\mathrm{N} 8-\mathrm{C} 23-\mathrm{C} 24-\mathrm{N} 9$
$108.5(15)$
$109.0(14)$
$105(2)$
$112(2)$
$110(2)$
$178.55(18)$
$-1.15(19)$
$179.60(15)$
$0.52(18)$
$-0.1(3)$
$-179.23(15)$
$179.48(17)$
$-0.8(2)$
$1.29(19)$
$-175.17(18)$
$-1.5(2)$
$175.21(16)$
$0.5(3)$
$179.87(17)$
$-0.7(2)$
$0.2(3)$
$-179.37(18)$
$0.3(3)$
$-0.8(3)$
$0.3(3)$
$-0.3(3)$
$0.0(3)$
$0.2(3)$
$-178.27(17)$
$6.0(3)$
$-6.7(3)$
$177.37(17)$
$0.1(3)$
$0.1(3)$
$-8.3(3)$
$175.69(17)$

Hydrogen-bond geometry $\left(A,{ }^{\circ}\right)$

\begin{tabular}{lllll}
\hline$D-\mathrm{H} \cdots A$ & $D-\mathrm{H}$ & $\mathrm{H} \cdots A$ & $D \cdots A$ & $D-\mathrm{H} \cdots A$ \\
\hline $\mathrm{O} 1-\mathrm{H} 1 A \cdots \mathrm{Cl} 4^{\mathrm{i}}$ & $0.82(2)$ & $2.63(2)$ & $3.4431(15)$ & $168(3)$ \\
$\mathrm{O} 1-\mathrm{H} 2 B \cdots \mathrm{Cl} 2$ & $0.81(2)$ & $2.36(2)$ & $3.1598(18)$ & $169(3)$ \\
$\mathrm{O} 2-\mathrm{H} 2 C \cdots \mathrm{Cl} 3$ & $0.82(2)$ & $2.33(2)$ & $3.1244(17)$ & $165(2)$ \\
$\mathrm{O} 2-\mathrm{H} 2 D \cdots \mathrm{Cl} 1$ & $0.81(1)$ & $2.64(1)$ & $3.4492(15)$ & $171(3)$ \\
$\mathrm{O} 3-\mathrm{H} 3 D \cdots \mathrm{Cl} 5^{\mathrm{ii}}$ & $0.80(2)$ & $2.69(2)$ & $3.4586(15)$ & $164(3)$ \\
$\mathrm{O} 3-\mathrm{H} 3 E \cdots \mathrm{Cl} 6^{\mathrm{ii}}$ & $0.82(2)$ & $2.32(2)$ & $3.1364(18)$ & $173(3)$
\end{tabular}




$\begin{array}{lllll}\mathrm{N} 1-\mathrm{H} 1 \cdots \mathrm{O} 1 & 0.83(2) & 1.92(2) & 2.746(2) & 174(2) \\ \mathrm{N} 2-\mathrm{H} 2 \cdots \mathrm{Cl} 1^{\mathrm{iii}} & 0.71(2) & 2.44(2) & 3.1519(17) & 175(2) \\ \mathrm{N} 3-\mathrm{H} 3 \cdots \mathrm{Cl} 3 & 0.88(3) & 2.30(3) & 3.105(2) & 153(2) \\ \mathrm{N} 3-\mathrm{H} 3 A \cdots \mathrm{Cl} 1 & 0.86(4) & 2.26(3) & 3.119(2) & 173(3) \\ \mathrm{N} 3-\mathrm{H} 3 B \cdots \mathrm{Cl} 4^{\text {iv }} & 0.99(3) & 2.33(2) & 3.267(2) & 156.8(19) \\ \mathrm{N} 4-\mathrm{H} 4 A \cdots \mathrm{O} 2 & 0.85(2) & 1.91(2) & 2.754(2) & 171(2) \\ \mathrm{N} 5-\mathrm{H} 5 A \cdots \mathrm{Cl} 15 & 0.83(2) & 2.31(2) & 3.1205(17) & 165(2) \\ \mathrm{N} 6-\mathrm{H} 6 A \cdots \mathrm{Cl} 2 & 0.87(3) & 2.33(3) & 3.107(2) & 150(2) \\ \mathrm{N} 6-\mathrm{H} 6 B \cdots \mathrm{Cl} 1^{\text {v }} & 0.98(3) & 2.36(2) & 3.287(2) & 158.2(18) \\ \mathrm{N} 6-\mathrm{H} 6 C \cdots \mathrm{Cl} 4^{\mathrm{i}} & 0.79(3) & 2.35(4) & 3.1347(19) & 176(3) \\ \mathrm{N} 7-\mathrm{H} 7 \cdots \mathrm{O} 3 & 0.85(3) & 1.89(3) & 2.742(2) & 175(3) \\ \mathrm{N} 8-\mathrm{H} 8 \cdots \mathrm{Cl} 4 & 0.81(2) & 2.31(2) & 3.1049(17) & 172(2) \\ \mathrm{N} 9-\mathrm{H} 9 A \cdots \mathrm{Cl} 6 & 0.83(3) & 2.35(3) & 3.100(2) & 151(2) \\ \mathrm{N} 9-\mathrm{H} 9 B \cdots \mathrm{Cl} 15 & 0.80(3) & 2.32(2) & 3.120(2) & 176(2) \\ \mathrm{N} 9-\mathrm{H} 9 C \cdots \mathrm{Cl} 15^{\text {vi }} & 0.99(3) & 2.34(3) & 3.270(2) & 157(2) \\ \mathrm{C} 4-\mathrm{H} 4 \cdots \mathrm{Cl} 1^{\mathrm{i}} & 0.92(2) & 2.81(2) & 3.535(2) & 136.7(16) \\ \mathrm{C} 8-\mathrm{H} 8 B \cdots \mathrm{Cl} 3^{\text {vii }} & 0.93(3) & 2.65(3) & 3.544(2) & 162(2) \\ & & & & \end{array}$

Symmetry codes: (i) $x, y-1, z$; (ii) $-x,-y+1,-z+1$; (iii) $-x+3,-y,-z+2$; (iv) $x+1, y-1, z$; (v) $x-1, y, z$; (vi) $-x+1,-y+1,-z+1$; (vii) $-x+2,-y,-z+2$. 